\title{
Infrared Imaging of Planetary Nebulae from the Ground Up
}

\author{
Joseph L. Hora \\ Harvard-Smithsonian Center for Astrophysics, 60 Garden St., MS-65, Cambridge, MA \\ 02138-1516, USA \\ email: jhora@cfa.harvard.edu
}

\begin{abstract}
New ground-based telescopes and instruments, the return of the NICMOS instrument on the Hubble Space Telescope (HST), and the recent launch of the Spitzer Space Telescope have provided new tools that are being utilized in the study of planetary nebulae. Multiwavelength, high spatial resolution ground-based and HST imaging have been used to probe the inner regions of young PNe to determine their structure and evaluate formation mechanisms. Spitzer/IRAC and MIPS have been used to image more evolved PNe to determine the spatial distribution of molecular hydrogen, ionized gas, and dust in the nebulae and halos.
\end{abstract}

Keywords. Spitzer Space Telescope, infrared: ISM, ISM: molecules, planetary nebulae: general, techniques: high angular resolution

\section{Introduction}

Infrared imaging has been an important observational technique for planetary nebulae (PNe) for several reasons. First, the ejected matter from the star often obscures the inner regions of the nebula. In the infrared, one can penetrate the surrounding dust and gas and see into the regions near the central star, which may be key in understanding the formation mechanisms that lead to the PN structure. Second, there are many infrared lines that are important tracers and diagnostics of shocks and photodissociation regions (PDRs). These include the bright $\mathrm{H}_{2}$ line at $2.12 \mu \mathrm{m}$ and many other lines from $2-28 \mu \mathrm{m}$, the $[\mathrm{Fe}$ II $] 1.64 \mu \mathrm{m}$ line, and several other bright diagnostic lines such as $4.05 \mu \mathrm{m} \mathrm{Br} \alpha$, $12.81 \mu \mathrm{m}[\mathrm{Ne} \mathrm{II}]$, and other lines. Finally, there are components of the nebula that can only be observed in the infrared. These include the PAH emission features at 3.3, 6.2, 7.7, 8.6, and $11.2 \mu \mathrm{m}$, and thermal continuum emission from hot, warm, or cool dust.

Since the last PNe symposium, observers have made use of improved facilities and instruments to probe $\mathrm{PNe}$ in the infrared. There are now several telescopes in the 8 $10 \mathrm{~m}$ class in both hemispheres that provide a significant increase in sensitivity and resolution over what was previously available. The capability of the instruments on these telescopes has also been greatly enhanced with large-format IR imagers, many with adaptive optics (AO) that can deliver nearly diffraction-limited image quality at longer wavelengths. These systems utilize narrow-band filters to isolate spectral features and nearby continuum wavelengths to map the emission components of the nebula.

Previous and current space missions have also provided new resources for infrared imaging. Surveys such as the MSX survey of the Galactic Plane, and the all-sky 2MASS survey are important datasets that are being utilized to find or study PNe in the infrared. The HST/NICMOS instrument has been used to study the compact structure of young PNe in the near-IR. Finally, the Spitzer Space Telescope (Werner et al. 2004) was launched in August 2003 with three instruments that have imaging capability from $3-200 \mu \mathrm{m}$. 
In this review I will summarize some representative published results since the last IAU Symposium, and some of the Spitzer GTO observations of PNe. Although I stress here the infrared imaging aspects of recent results, the IR emission is only one important part of the full picture of these PNe. Many investigators employ a multi-wavelength approach using images and spectra that greatly enhances the understanding of the PNe's structure.

\section{Ground-based Near-Infrared (1-5 $\mu \mathrm{m})$ Imaging}

Early near-IR array camera imaging surveys (e.g., Latter et al. 1995; Kastner et al. 1996) focused on narrowband imaging of various well-studied PNe, exploring the relationship between $\mathrm{H}_{2}$ emission and morphological type, and examining differences between the near-IR and optical structure. Much of the recent ground-based near-IR imaging has concentrated on high angular resolution narrowband observations of young PNe. The goal has been to probe the compact structures near the core and in the lobes of the PNe to gain an understanding of the mechanisms that are creating the bipolar or asymmetric morphology.

One example is Volk et al. (2004) who used the Gemini telescope with the QUIRC instrument and Hokupa'a AO system to perform high resolution (0.15") $2.12 \mu \mathrm{m}$ and narrowband continuum imaging of three young PNe, IRAS $19306+1407$, IRAS $20028+3910$, and IRAS $22023+5249$. They found the $\mathrm{H}_{2}$ emission primarily in the lobes or in an equatorial torus around the star. They demonstrated that with ground-based near-IR imaging one can detect and resolve structures in objects that are in the very early stages of PN formation.

Sahai et al. (2005) applied similar techniques to another young PN, IRAS 16342 - 3814 . They used the Keck II telescope with NIRC2 and natural guide star AO system to obtain $H, K_{p}, L_{p}$, and $M_{s}$ images that show limb-brightened bipolar lobes with a "corkscrew" pattern inscribed in the walls of the lobes. They conclude that this pattern is the result of a well-collimated jet with a diameter $\leqq 100 \mathrm{AU}$ and a precession period $\leqq 50 \mathrm{yr}$.

Ground-based near-IR imaging has also been used in conjunction with HST (and longer wavelength) imaging to probe regions that are heavily obscured near the core and to trace the distribution of PAH molecules and hot dust emission. Matsuura et al. (2005a) used the ISAAC instrument on the VLT to image the bipolar PN NGC 6302 in the $3.28 \mu \mathrm{m}$ $\mathrm{PAH}$ and $\mathrm{Br} \alpha$ lines, as well as continuum wavelengths. The PAH feature emission is found to be similar to the ionized emission, although in the PAH image there is an additional local maximum in a "spur" of emission and a shell extending away from the core. They also used the JCMT to obtain a $450 \mu \mathrm{m}$ continuum image which shows an extended massive dust disk around the central core. From these data, they were able to estimate the mass of the dust and the disk, examine the complicated structure in the core, and detect a point source that may be related to the central star. Matsuura et al. (2005b) performed a similar study of the bipolar PN NGC 6537.

The observations by Smith (2003) of Menzel 3 (Mz 3) show the power of combining narrowband images with optical and near-IR spectra. $\mathrm{Mz} 3$ is a bipolar nebula that is similar in many respects to $\mathrm{M} 2-9$, except that $\mathrm{Mz} 3$ is a bipolar with no detected $\mathrm{H}_{2}$ emission. With the spectra, Smith used the CLOUDY program to model the central source temperature and determine abundances of the ejecta. The images in particular lines indicate how conditions vary across the nebula.

When high spectral resolution imaging can be performed, a wealth of information can be obtained on the morphology and kinematics of the nebula. This has been demonstrated for example in the PN NGC 7027 and AFGL 618 (Cox et al. 2002; Cox et al. 2003). They used the BEAR instrument, an imaging Fourier Transform Spectrometer operated at the 
CFHT that provided a spectral resolution of $\sim 9 \mathrm{~km} \mathrm{~s}^{-1}$. These data allowed Cox et al. to examine the kinematics of the ionized gas and molecular components, and to probe the interactions in the outflows.

\section{Ground-based Mid-Infrared (8-25 $\mu \mathrm{m})$ Imaging}

The recent availability of facility mid-IR instruments on large telescopes has enhanced the ability to probe even deeper into obscured regions, and to detect emission from warm dust and molecules. The objects which have been most often observed are young PNe which are bright in the mid-IR and their central regions are obscured by dust in the optical. The first instruments based on sensitive mid-IR array detectors were developed in the late 1980's and were used on the large IR-optimized telescopes of that time to study this class of objects (e.g., Hora et al. 1990; Meixner et al. 1993; Deutsch et al. 1993; Persi et al. 1994; Kömpe et al. 1997; Meixner et al. 1999). Some mid-IR imaging has continued to be performed on 3-4 m telescopes. For example, Kemper et al. (2002) used TIMMI2 on the ESO 3.6m to image NGC 6302, and Matsuura et al. (2004) imaged the post-AGB star IRAS 16279-4757. Smith \& Gehrz (2005) used MIRLIN on the IRTF to image M2-9, Mz 3, and He 2-104.

Recently, mid-IR instruments on Keck, the VLT, and Gemini telescopes have been exploited to achieve higher sensitivity and resolution. Muthumariappan et al. (2006) used the Gemini South telescope with the T-ReCS instrument to image Hen 3-401, a bipolar nebula viewed nearly edge-on, with lobes separated by a dust lane. With continuum images at 10.4 and $18.3 \mu \mathrm{m}$, they constructed a color temperature map of the emission in the lobes. They obtained a narrowband image in the $11.3 \mu \mathrm{m}$ PAH feature and determined that the PAH emission is organized in concentric sets of arcs in the lobes, which suggests several ejection epochs. In another investigation of mid-IR spectral features, Kwok et al. (2002) used the Gemini North telescope and OSCIR to examine two proto-PN that possess the $21 \mu \mathrm{m}$ emission feature. They found a similar morphology in the 11.3 and $21 \mu \mathrm{m}$ emission, suggesting that the carriers of both features originated in the material ejected during the AGB phase.

In another example of a multi-wavelength approach, Lagadec et al. (2006) used archive HST optical data and near- and mid-IR data from $3-13 \mu \mathrm{m}$ to examine the core of the PN Hen 2-113. This PN had been imaged previously by Sahai et al. (2000) who found the morphology to be bipolar, with two ring-like structures around the central star location. Lagadec et al. obtained their images with several instruments (NACO, MIDI, ISAAC, and TIMMI) at ESO, and analyzed them in conjunction with the HST data. They determined that the PN has a "diabolo" structure, with the previously observed rings being the opening of two cone-like structures defining the bipolar outflow, and a dark lane between them. There are also many other structures detected, such as bright regions and spots, and filaments and holes in other parts of the lobes. The distribution of the PAH emission leads them to conclude that the PAHs formed more recently than the continuum-emitting dust.

\section{HST/NICMOS Imaging}

The installation of the NICMOS instrument on HST in 1997 provided the capability for high sensitivity, diffraction-limited 1-2.5 $\mu \mathrm{m}$ imaging with many narrow and broad filters, and was used for several studies of PNe (e.g., Sahai et al. 1998 (AFGL 2688); Latter et al. 2000 (NGC 7027)). The cryogen ran out prematurely early in 1999 rendering the instrument no longer functional. In early 2002, shortly after the last IAU PN Symposium, 
a cryocooler was installed that brought the NICMOS instrument back to operation. Since then, several new projects have been carried out, as well as studies of data in the archive that were obtained during the original cryogen lifetime.

One example of new NICMOS imaging that was performed on a young PN is Sahai et al. (2005), who imaged the OH/IR star IRAS 19024+0044 with NICMOS (1.1 and $1.6 \mu \mathrm{m}$ ) and the ACS (at 0.6 and $0.8 \mu \mathrm{m}$ ). They found a compact multipolar PN with at least six elongated, limb-brightened lobes originating from the central star. The central region is brighter at the longer wavelengths, with the peak in the $1.6 \mu \mathrm{m}$ image positioned near the center of symmetry of the lobes. The NICMOS images reveal the region near the core where there are dark lanes in the optical images.

Another result from new NICMOS imaging is the Meixner et al. (2005) study of several regions in NGC 7293 (the Helix). While the ACS was imaging the central region and main ring, NICMOS was used in parallel to image positions on the main ring and the halo. In this nearby $\mathrm{PN}$, they resolved the $\mathrm{H}_{2}$ emission in the ring into individual molecular knots that have the appearance of small arcs with their apex pointing toward the central star. The arcs are similar to the cometary knots previously observed in the central hole, but without the long tails. At increasing radius, they found the $\mathrm{H}_{2}$ emission becomes less structured. From the $\mathrm{H}_{2}$ images, they estimate a total neutral gas mass of the PN.

\section{IR Surveys (2MASS, MSX)}

Infrared imaging surveys such as 2MASS and MSX have become available since the last IAU PNe symposium and have provided a resource that has begun to be exploited for the study of PNe. For example, Phillips \& Ramos Larios have in a series of papers (Phillips \& Ramos Larios 2005; Ramos Larios \& Phillips 2005; Phillips \& Ramos Larios 2006) used the 2MASS database to study the near-IR properties of the PNe in the extended source catalog, and to examine hot dust in these nebulae. They report the presence of hot dust halos for several of the PNe with large $K$ and $H$ band excesses, possibly due to small grains. Gauba et al. (2003) used optical photometry along with 2MASS, MSX, and IRAS data to examine a sample of hot post-AGB candidates. They modeled the SEDs and derived mass loss rates, temperatures, distances, and sizes of the dust envelopes.

\section{Spitzer/IRAC and MIPS Imaging}

With the launch of the Spitzer Space Telescope, an important new tool became available for the study of evolved stars and their ejecta. The three instruments on board Spitzer IRAC (Fazio et al. 2004), IRS (Houck et al. 2004) and MIPS (Rieke et al. 2004) provide imaging capability from 3-200 $\mu \mathrm{m}$. The IRS is a powerful spectrograph operating in the 5-40 $\mu \mathrm{m}$ range, and is reviewed in another paper at this symposium (Bernard-Salas, these proceedings). In this review I focus on Spitzer's broad-band imaging capability at $3.6,4.5,5.8,8,24,70$, and $160 \mu \mathrm{m}$. The instruments sample a spectral range that is difficult or impossible to observe from the ground, and can achieve $\mu \mathrm{Jy}$ sensitivities in a matter of minutes over large fields, making Spitzer the most sensitive platform ever for probing faint compact mid-IR sources and extended emission from PNe. The relatively low spatial resolution $(\sim 2$ " for IRAC) means that Spitzer is complementary to groundbased instruments that have been used to resolve bright, compact structures.

\subsection{Spitzer Survey Data}

There are several large surveys already in the Spitzer public archive that can be used for the study of PNe. One of the largest is the GLIMPSE project (Churchwell et al. 2004) 

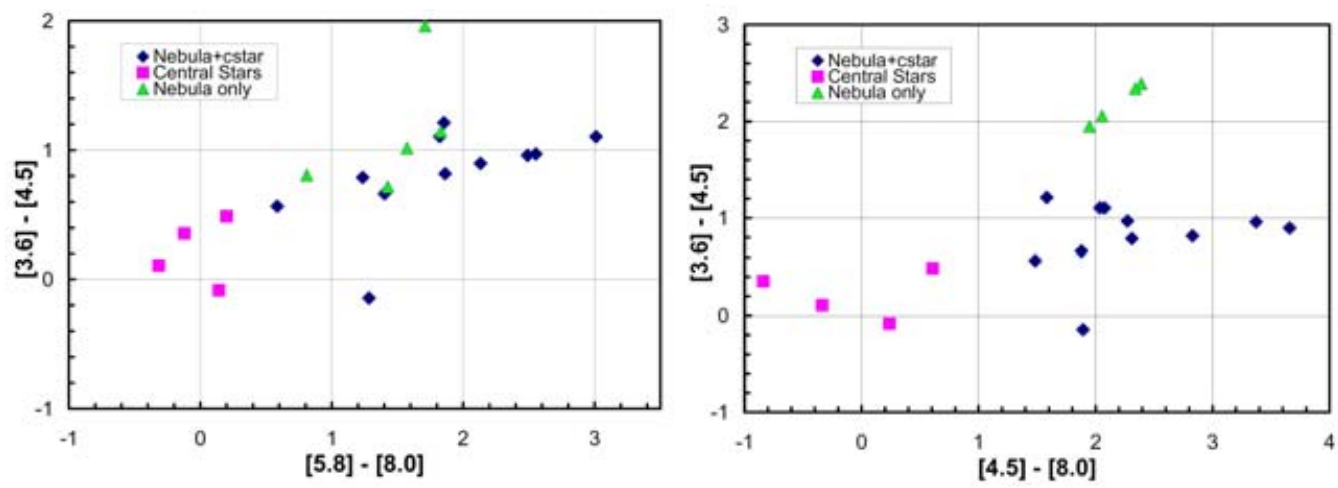

Figure 1. IRAC color-color plots for some of the PNe in the GTO sample. The left plot shows the 4 -band color-color plot, the plot on the right shows the plot with bands 1,2 , and 4 . The nebulae are red (brighter in bands 3 and 4) compared to main sequence stars and the PN central stars. This is likely due to emission in bands 3 and 4 from $\mathrm{H}_{2}$ or forbidden line emission from the ionized gas. In some cases where the central star was detected, the star and nebula were plotted separately.

which surveyed a large part of the galactic plane with IRAC. The MIPSGAL project (Carey et al. 2005) will map a similar area with the MIPS 24 and $70 \mu \mathrm{m}$ bands. The properties of known PNe can be determined, and also searches for new nebulae performed (e.g., Cohen et al. 2005). Another potentially useful data set is the SAGE survey of the LMC (Meixner et al. 2006), which imaged a $7 \times 7$ degree region with MIPS and IRAC. Source catalogs and mosaics for SAGE are expected to be released in late 2006. All data can be accessed via the Spitzer web site at http://ssc.spitzer.caltech.edu.

\subsection{IRAC survey of PNe}

The IRAC GTO program includes a project to image 35 PNe with IRAC. A parallel program was carried out in MIPS GTO time by W. Latter. Some early results have been presented (Hora et al. 2004; Hora et al. 2005; Hora et al. 2006), a sample are shown here. Figure 1 shows the IRAC colors for a number of PNe observed. The nebular colors are in general red, especially comparing the 3.6 or $4.5 \mu \mathrm{m}$ bands to the 5.8 and $8 \mu \mathrm{m}$.

The IRAC image of the Helix is shown in Figure 2 (Hora et al. 2006). The four bands are mapped into colors of blue to red in order of wavelength. The IRAC images and IRS spectra, along with $2.12 \mu \mathrm{m}$ images, confirm the results of Cox et al. (1998) who mapped the Helix with $I S O$ and found that the mid-IR emission was dominated by lines of $\mathrm{H}_{2}$. Along with Cox et al. and Meixner et al. (2005) we find that the $\mathrm{H}_{2}$ line ratios are inconsistent with PDR models. We find that they can be fit by models of shock-excited $\mathrm{H}_{2}$, with a small PDR component. The IRAC emission is clumpy in the rings, each clump appearing as a small arc pointing back toward the central star, as in the $2.12 \mu \mathrm{m} \mathrm{H}_{2}$ images. Outside of the ring, there are radial rays extending into the halo.

Figure 3 shows the IRAC images of the Dumbbell (NGC 6853) and the Ring nebula (NGC 6720). For the Dumbbell nebula, the appearance in the IRAC image is drastically different than in the optical. The IRAC emission is primarily from the roughly N-S dense equatorial region, and is dominated by the clumpy emission and radial rays that are strongest in the long wavelength channels. The appearance at $8 \mu \mathrm{m}$ is almost identical to the $2.12 \mu \mathrm{m} \mathrm{H}_{2}$ image, indicating a common origin. In the Ring nebula, there are similarities to the optical appearance, but the $\mathrm{H}_{2}$ emission in the IRAC bands highlights different parts of the nebular structure (see also Speck et al. 2003). In the IRAC color image in Figure 3, the central region is slightly green from emission in the $4.5 \mu \mathrm{m}$ 


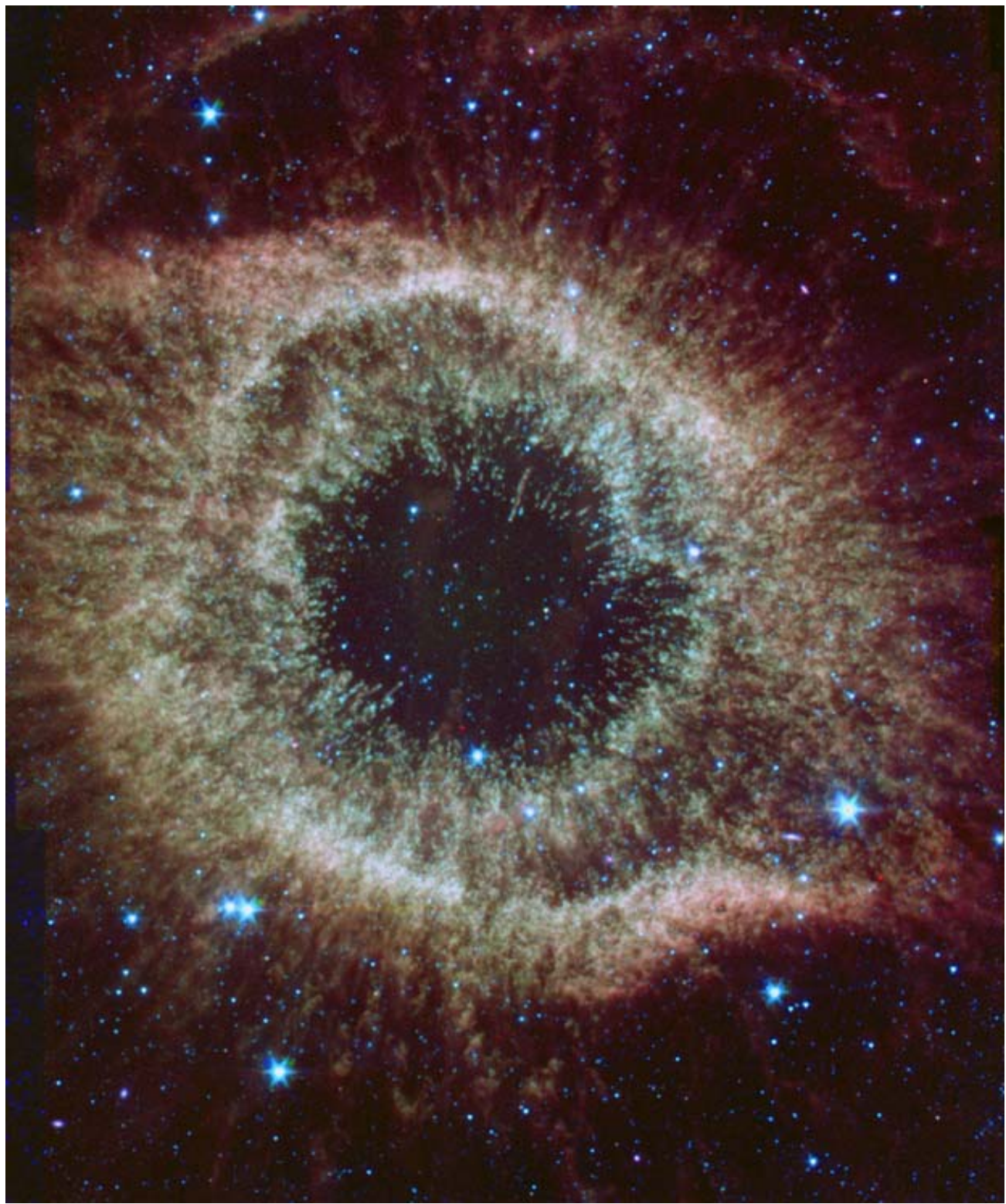

Figure 2. IRAC image of the Helix, with the 3.6, 4.5, 5.8, and $8.0 \mu \mathrm{m}$ bands are mapped to blue, green, orange, and red, respectively. The image is approximately $24 \times 26$ arcmin in size. The cometary knots are visible inside the main ring; the knot tips are relatively brighter in the 3.6 and $4.5 \mu \mathrm{m}$ bands, and the tails are brighter at $8 \mu \mathrm{m}$. The nebular emission is dominated by $\mathrm{H}_{2}$ in the IRAC bands.

bandpass, possibly due to atomic lines such as $\operatorname{Br} \alpha,[\mathrm{Mg} \mathrm{IV}]$ and $[\mathrm{Ar} \mathrm{VI}]$ in that band. The edge of the ring and the outer halo are orange-red, consistent with emission from $\mathrm{H}_{2}$ in the 5.8 and $8 \mu \mathrm{m}$ bands.

An early MIPS imaging result was reported by $\mathrm{Su}$ et al. (2004), who imaged NGC 2346 at 24,70 , and $160 \mu \mathrm{m}$. The distribution of the $24 \mu \mathrm{m}$ emission was found to be very similar to the optical $\mathrm{H} \alpha$ emission, except for a hot dust component that peaks at the 

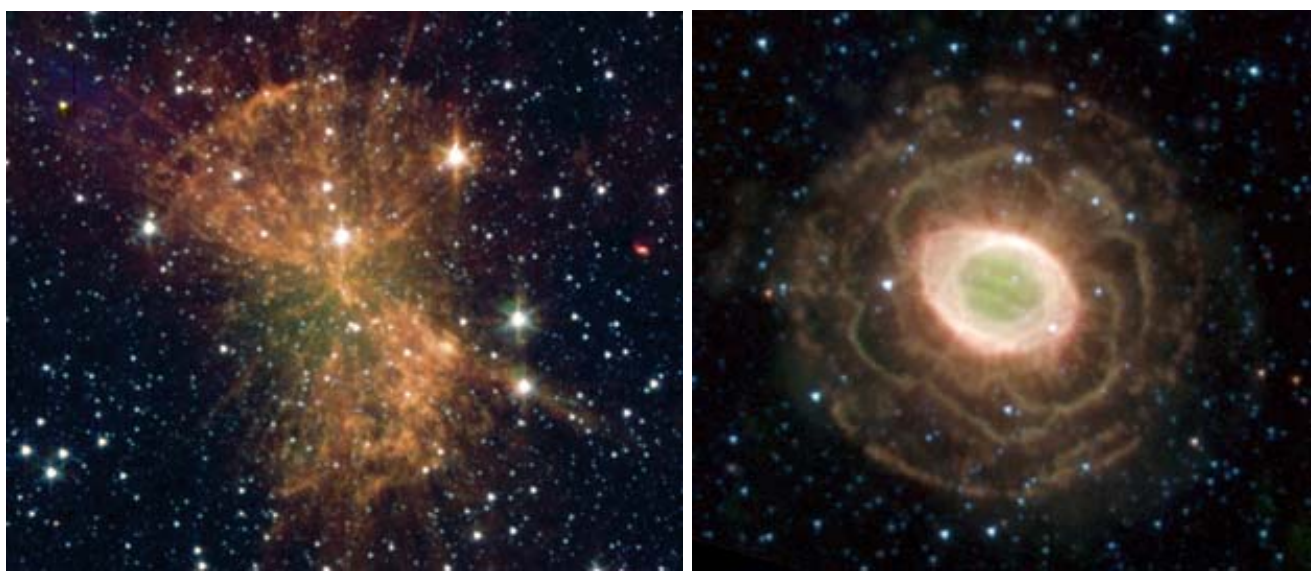

Figure 3. IRAC color images of PNe. The 3.6, 4.5, 5.8, and $8.0 \mu \mathrm{m}$ bands are blue, green, orange, and red, respectively. Left: the Dumbbell (NGC 6853). Right: the Ring (NGC 6720).

position of the central star. This dust is likely responsible for the previously observed deep fadings of the central star. The $70 \mu \mathrm{m}$ image shows a double-peaked morphology in the narrow waistband of the nebula, indicating a possible dust torus structure. The 160 $\mu \mathrm{m}$ image shows that the nebula is contained in an extended cold dust envelope.

\section{Conclusions}

Infrared imaging is an important probe of the molecular and dust components of $\mathrm{PNe}$, as well as the ionized gas in regions that are obscured at shorter wavelengths. Much of the recent work has focused on high spatial resolution studies of young PNe, exploring the early development of nebula formation and searching for evidence of the processes responsible for the nebula morphology. New surveys and instruments such as those on board Spitzer have recently become available and are being utilized to expand our knowledge of the formation and evolution of PNe.

\section{Acknowledgements}

This work is based in part on observations made with the Spitzer Space Telescope, which is operated by the Jet Propulsion Laboratory, California Institute of Technology under NASA contract 1407. Support for the IRAC instrument was provided by NASA under contract number 960541 issued by JPL.

\section{References}

Carey, S. J. et al. 2005, AAS, 207, 6333

Churchwell, E. B., et al. 2004, ApJS, 154, 322

Cohen, M., Green, A. J., et al. 2005, ApJ, 627, 446

Cox, P., et al. 1998, ApJ, 495, L23

Cox, P., Huggins, P. J., Maillard, J.-P., Habart, E., Morisset, C., Bachiller, R., \& Forveille, T. 2002, A\&BA, 384, 603

Cox, P., Huggins, P. J., Maillard, J.-P., Muthu, C., Bachiller, R., \& Forveille, T. 2003, ApJ, 586, L87

Deutsch, L. K., Hora, J. L., Hoffmann, W. F., Fazio, G. G. \& Shivanandan, K. 1993, IAU Symp. 155, Planetary Nebulae, eds. R. Weinberger \& A. Acker (Dordrecht: Kluwer), 344

Fazio, G. G. et al. 2004, ApJS, 154, 10 
Gauba, G., Parthasarathy, M., Kumar, B., Yadav, R. K. S., \& Sagar, R. 2003, A\&3A, 404, 305

Hora, J. L., Deutsch, L. K., Hoffmann, W. F., \& Fazio, G. G. 1990, ApJ, 353, 549

Hora, J. L., Latter, W. B., Allen, L. E., Marengo, M., Deutsch, L. K., \& Pipher, J. L. 2004, ApJS, 154, 296

Hora, J. L., Latter, W. B., Marengo, M., Fazio, G. G., Allen, L. E. \& Pipher, J. L. 2005, AAS, 206,3901

Hora, J. L., Latter, W. B., Smith, H. A., \& Marengo, M. 2006, in press (astro-ph/0607541)

Houck, J. et al. 2004, ApJS, 154, 18

Kastner, J. H., Weintraub, D. A., Gatley, I., Merrill, K. M., \& Probst, R. G. 1996, ApJ, 462, 777

Kemper, F., Molster, F. J., Jäger, C., \& Waters, L. B. F. M. 2002, A\&A, 394, 679

Kömpe, C., Lehmann, T., Gürtler, J., Stecklum, B., \& Krügel, E. 1997, IAU Symp. 180, Planetary Nebulae, eds. H. J. Habing \& H. J. G. L. M. Lamers (Dordrecht: Kluwer), 352

Kwok, S., Volk, K., \& Hrivnak, B. J. 2002, ApJ, 573, 720

Lagadec, E., Chesneau, O., Matsuura, M., De Marco, O., de Freitas Pacheco, J. A., Zijlstra, A. A., Acker, A., Clayton, G. C., \& Lopez, B. 2006, A\&\&A, 448, 203

Latter, W. B., Kelly, D. M., Hora, J. L., Deutsch, L. K. 1995, ApJS, 100, 159

Latter, W. B., Dayal, A., Bieging, J. H., Meakin, C., Hora, J. L., Kelly, D. M., Tielens, A. G. G. M 2000, ApJ, 783, 539

Matsuura, M., Zijlstra, A. A., Molster, F. J., Hony, S., Waters, L. B. F. M., Kemper, F., Bowey, J. E., Chihara, H., Koike, C., Keller, L. P. 2004, ApJ, 604, 791

Matsuura, M., Zijlstra, A. A., Molster, F. J., Waters, L. B. F. M., Nomura, H., Sahai, R., \& Hoare, M. G. 2005a, MNRAS, 359, 383

Matsuura, M., Zijlstra, A. A., Gray, M. D., Molster, F. J., \& Waters, L. B. F. M. 2005b, MNRAS, 359,383

Meixner, M., Skinner, C. J., Temi, P., Rank, D., Bregman, J., Ball, J. R., Keto, E., Arens, J. F., Jernigan, J. G. 1993, ApJ, 411, 266

Meixner, M., Ueta, T., Dayal, A., Hora, J. L., Fazio, G., Hrivnak, B, J., Skinner, C. J., Hoffmann, W. F., \& Deutsch, L. K. 1999, ApJS 122, 221

Meixner, M., McCullough, P., Hartman, J., Son, M., \& Speck, A. 2005, AJ, 130, 1784

Meixner, M. et al. 2006, AJ, in press (astro-ph/0606356)

Muthumariappan, C., Kwok, S., \& Volk, K. 2006, ApJ, 640, 353

Persi, P., Ferrari-Toniolo, M., Marenzi, A. R., Busso, M., Corcione, L., Nicolini, G., Shivanandan, K., 1994, Exp. Astr., 3, 171

Phillips, J. P. \& Ramos Larios, G. 2005, MNRAS, 364, 849

Phillips, J. P. \& Ramos Larios, G. 2006, MNRAS, 368, 1773

Ramos Larios, G., \& Phillips, J. P. 2005, MNRAS, 357, 732

Rieke, G. H. et al. 2004, ApJS, 154, 25

Sahai, R., Hines, D. C., Kastner, J. H., Weintraub, D. A., Trauger, J. T., Rieke, M. J., Thompson, R. I., \& Schneider, G. 1998, ApJ, 492, L163

Sahai, R., Nyman, L.-A., \& Wootten, A. 2000, ApJ, 543, 880

Sahai, R., Le Mignant, D., Sanchez Contreras, C., Campbell, R. D., \& Chaffee 2005, ApJ, 622, L53

Smith, N. 2003, MNRAS, 342, 383

Smith, N., \& Gehrz, R. D. 2005, AJ, 129, 969

Speck, A. K., Meixner, M., Jacoby, G. H., \& Knezek, P. M. 2003, PASP, 115, 170

$\mathrm{Su}, \mathrm{K}$. Y. L., et al. 2004, ApJS, 154, 302

Volk, K., Hrivnak, B. J., \& Kwok, S. 2004, ApJ, 616, 1181

Werner. M. W. et al., 2004, ApJS, 154, 1 